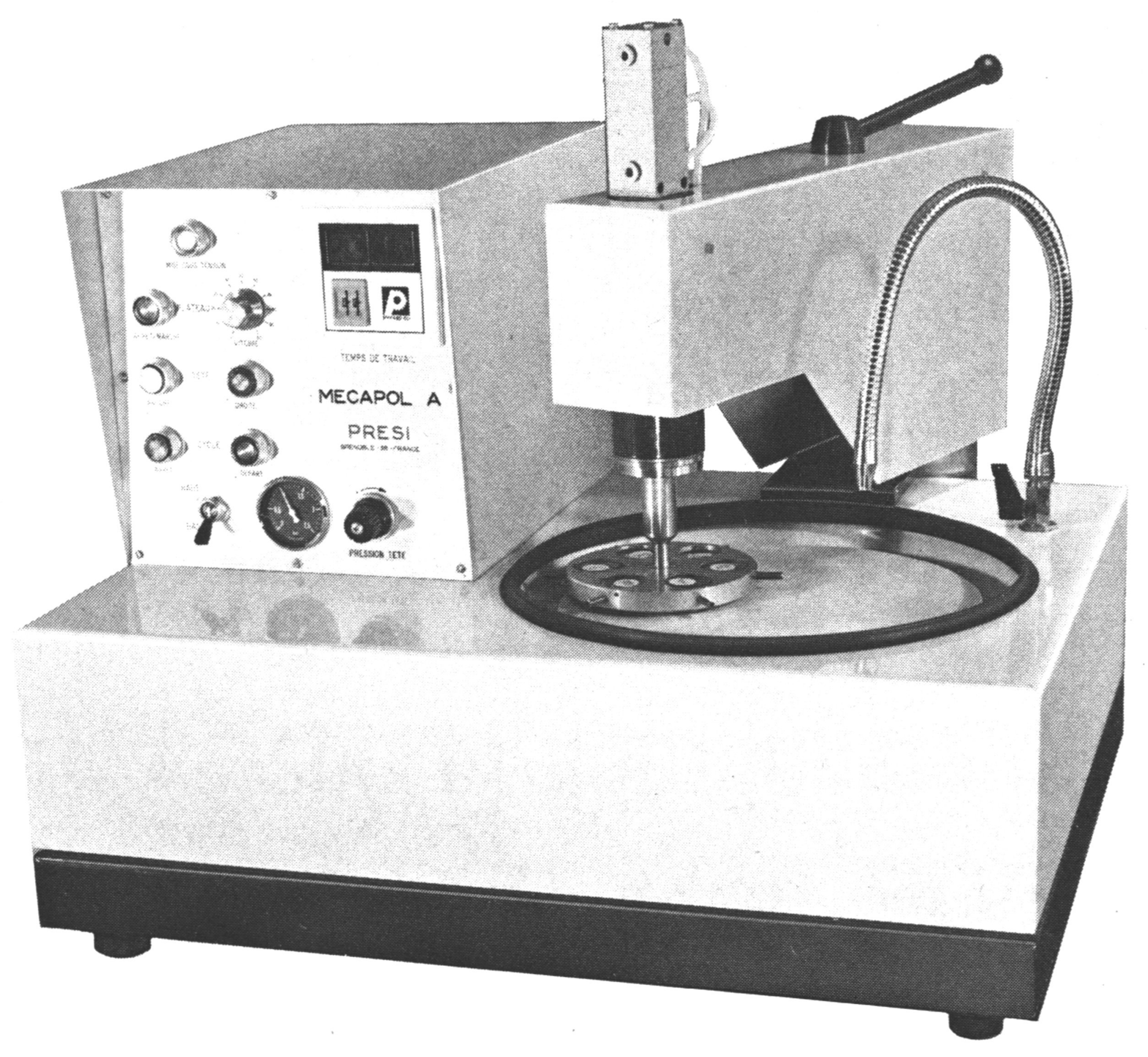

\section{Mit PRESI-}

Maschinen bessere Ergebnisse

Unser Prinzip:

Sachliche Voraussetzung für beste Leistung.

Solide Konstruktion

für lange Lebensdauer.

Optimale Sicherheit

für besten Arbeitsschutz.

\section{WIR BIETEN DAS OPTIMALE PREISLEISTUNGS- VERHÄLTNIS}

\section{Automatische Schleif- und Poliermaschine MECAPOL A}

Starker Antriebsmotor (zwei Geschwindigkeiten oder stufenlos auf Wunsch).

Schnellwechselplatten $\varnothing 300 \mathrm{~mm}$. • Automatischer Probenhalter mit Motor mit zwei Dreh-Richtungen. Automatisch regulierbarer Druck von $0-50 \mathrm{~kg}$. Probenhalterplatten für 12, 8, 6 oder 4 Proben von $\varnothing 30 \mathrm{~mm}$ bis $\varnothing 50 \mathrm{~mm}$.

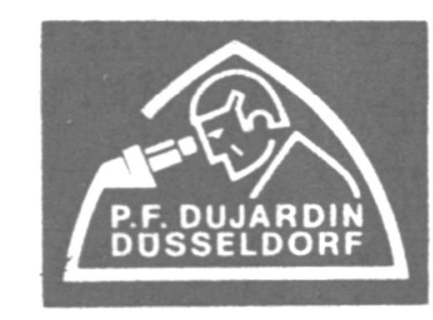

ein Begriff in der Metallographie

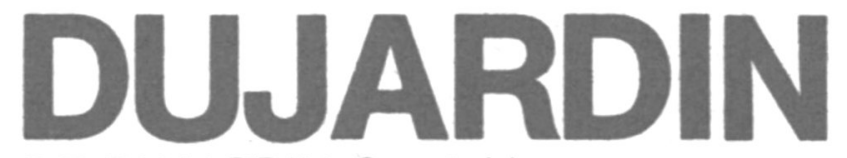

P.F. DUJARDIN G.m.b.H.

Ringbahnstraße 1

4040 Neuss 1

Telefon (02101) 275058, Telex 8518034

General Vertreter für Deutschland von:

PRESI-DUJARDIN stehen Ihnen gemeinsam mit 100 Jahren Metallographieerfahrung zur Verfügung.

PRESI Poisat-Grenoble/France 


\section{UNIVERSITÄT

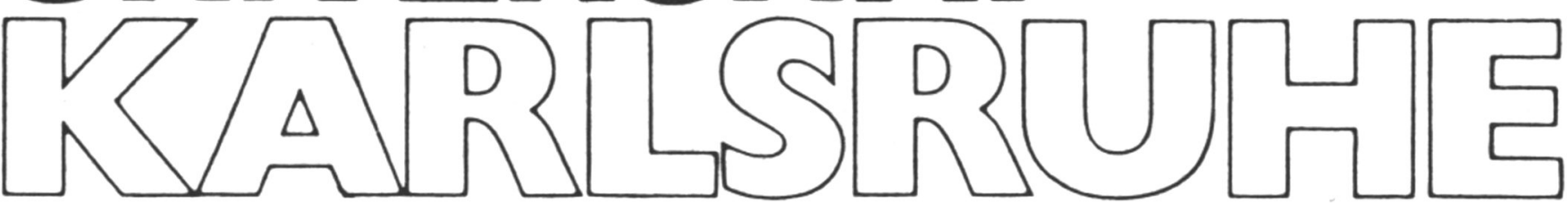

Das Institut für Werkstoffkunde II sucht zum 1.4.1984 eine/n

\section{Metallograph/in oder \\ physikal.-techn. Assistent/in}

Das Aufgabengebiet umfaßt die Mitarbeit an Forschungsvorhaben auf den Gebieten der technischen Keramik und der pulvermetallurgisch hergestellten Stähle, der metallographischen und keramographischen Präparation und Beurteilung der Werkstoffe, der Werkstoffprüfung sowie der Auswertung und Dokumentation von Versuchsergebnissen.

Der Arbeitsplatz befindet sich auf dem Gelände des Kernforschungszentrums.

Schriftliche Bewerbungen mit den üblichen Unterlagen werden erbeten an die Universität Karlsruhe, Personalabteilung, Kaiserstraße 12, 7500 Karlsruhe. 


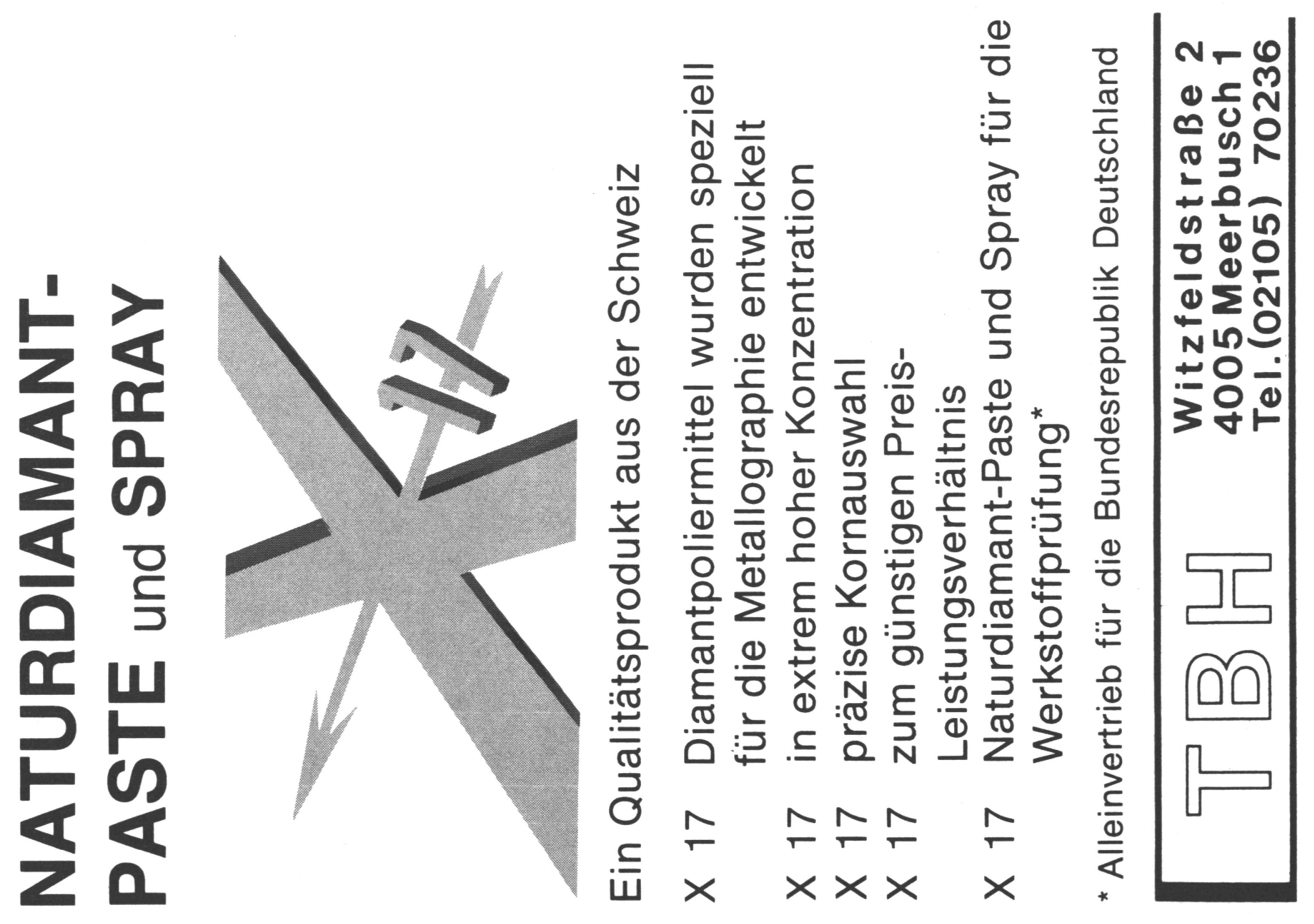


Praktische Metallographie

Herausgeber/Editor

Hauptschriftleiter/Editor in Chief

Redaktionssekretariat/Secretary

\section{Schriftleitung/Editorial Board}

Prof. Dr. H.-E. Bühler

Didier-Werke AG, Lessingstraße 16-18

D-6200 Wiesbaden

\section{Chris Bagnall}

Westinghouse Electric Corporation

Advanced Reactor Division

P. O. Box 158

Madison, PA 15663, USA

Dr. J. L. Chermant

Equipe Matériaux-Microstructure, L. A. 251

I.S.M.R.A.-Université, F-14032 Caen Cedex

Dr. G. Elssner

Max-Planck-Institut für Metallforschung

Seestraße 92, D-7000 Stuttgart 1

\section{R. J. Gray}

National Laboratory Oak Ridge

Oak Ridge, Tenn. 37830, USA

Prof. Dr. E. Hornbogen

Ruhr -Universität, Bochum

Postfach 2148, D-4630 Bochum

Dr. H. P. Hougardy

Max-Planck-Institut für Eisenforschung

Max-Planck-Straße 1, D-4000 Düsseldorf

Prof. Dr. F. Jeglitsch

Montanuniversität

A-8700 Leoben

Verlag und Anzeigenannahme/

Publisher and

Advertisement Contractor

Druck/Printing

Erscheinungsweise monatlich

Jährlicher Bezugspreis 1984 DM 139,-*

Einzelheft DM 16,-“ zzgl. Porto

Ohne schriftliche Genehmigung des Verlages dürfen weder Nachdruck, Übersetzungen, Photokopie, Mikrofilm noch Übernahme in Datenverarbeitungsanlagen bzw. deren Sprachen oder Informationssysteme irgendwelcher Art erfolgen. Fotomech. Vervielfält. nach Maßgabe des zw. dem Börsenv. des Dt. Buchhandels e. V. und dem Bundesverband der Dt. Industrie abgeschl. Rahmenabkommens gestattet. Abbestellungen nur bis spätestens 2 Mon. vor Ablauf des Kalender-Jahres.

In Zusammenarbeit mit der DGM.

\section{Practical}

Metallography

Prof. Dr. G. Petzow

Max-Planck-Institut für Metallforschung

Heisenbergstraße 5, D-7000 Stuttgart 80

Dr. H. E. Exner

Max-Planck-Institut für Metallforschung

Seestraße 92, D-7000 Stuttgart 1

Karin Exner

Amselweg 3, D-7250 Leonberg 7

Prof. Dr. Ursula Martius Franklin

University of Toronto

Toronto 5, Ontario, Canada

J. A. Nelson

Buehler Ltd.

41 Waukegan Road, Lake Bluff, IL 60044, USA

Prof. Dr. G. Ondracek

Universität und Kernforschungszentrum Karlsruhe Postfach 3640, D-7500 Karlsruhe

Dr. L. Samuels

Materials Research Labs.

POB 50, Ascot Vale, Victoria, Australia

Dr. H.-J. Schüller

Allianz-Zentrum für Technik $\mathrm{GmbH}$

Krausstraße 14, D-8045 Ismaning

Prof. Dr. Stickler

Universität Wien

Währingerstraße 42, A-1090 Wien

Fachstudiendirektorin Charlotte Wachau

Lette-Verein

Fabeckstraße 43, D-1000 Berlin 33

\section{Dr. Riederer-Verlag GmbH}

Postfach 447

D-7000 Stuttgart 1, Telefon (07 11) 639797

Badendruck GmbH, Karlsruhe

Issued monthly

Annual subscription rate 1984 DM 139,-* Single copy DM $16,-^{\star}$ plus delivery charges

All rights reserved. Without permissions in writing by the publisher neither reprints, translations, photoprints, microfilms, nor transmitting in computer-systems or Informationcenters in any way will be allowed. Reproduction by photography or xerox etc. for private use only. Cancellations of subscriptions not later than 2 months to the end of each current year.

In cooperation with the German Society for Metals 